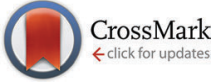

Cite this: New J. Chem., 2015, 39, 5171

Received (in Montpellier, France) 29th January 2015, Accepted 21st April 2015

DOI: 10.1039/c5nj00256g

www.rsc.org/njc

\section{Fluorosolvatochromism of furanyl- and thiophenyl-substituted acetophenones $\dagger$}

\author{
Nadine Friebe, ${ }^{a}$ Katja Schreiter, ${ }^{a}$ Joachim Kübel, ${ }^{\text {bc }}$ Benjamin Dietzek, ${ }^{\text {bc }}$ \\ Norbert Moszner, ${ }^{d}$ Peter Burtscher, ${ }^{d}$ Alexander Oehlke ${ }^{a}$ and Stefan Spange ${ }^{*^{a}}$
}

A series of para-substituted acetophenones bearing a furanyl or a thiophenyl moiety show a large Stokes-shift, which is a function of various solvent properties. Photophysical properties such as emission lifetime of the compounds have been determined using time-correlated-single photon counting to secure the intrinsic fluorescence behaviour. The solvent dependent position of the UV/Vis emission band $\tilde{\nu}_{\text {max,em }}$ of the compounds has been measured in 26 various solvents. The influence of the solvent on $\tilde{\nu}_{\text {max,em }}$ is of very complex nature and mathematically analysed by multiple square linear solvation energy (LSE)-correlation analysis using Catalán's four-solvent parameter set. Solvent acidity has a strong influence on the bathochromic shift of 2,5-disubstituted furan derivatives compared to the non-5-substituted furan and thiophene derivatives, which show a contrary behaviour. Therefore, the 5-cyanofuranyl-substituted acetophenone derivative is useful as a probe for measuring environmental properties by fluorescence spectroscopy.

\section{Introduction}

In recent years, there has been growing interest in organic fluorescent dyes because of their potential applications as fluorescent probes in chemistry, biochemistry and medicine, and in optoelectronics including organic light emitting diodes (OLEDs), sensors, and lasers. ${ }^{1-3}$ Such organic fluorophores can also act as environmental probes, e.g. acid-base, and solventsensitive indicators. ${ }^{4}$

In previous work, we have studied and discussed many solvatochromic dyes, which are suitable to measure environmental effects by shift of the position of the UV/Vis absorption band as a function of polarity of the environment. For this purpose, push-pull substituted catechol dyes, ${ }^{5 a}$ azobenzene chromophores being linked at polyvinylamine backbones, ${ }^{5 b}$ and specific aminobenzofurandione dyes as basicity probes for ionic liquids ${ }^{5 c}$ were used.

Interactions of solvatochromic dyes with pure solvents or solvent mixtures are determined by a combination of many effects. ${ }^{6}$ In order to separate the effects of specific interactions (hydrogenbonding capacity) from non-specific interactions including

\footnotetext{
${ }^{a}$ Department of Polymer Chemistry, Institute of Chemistry, Technische Universität Chemnitz, 09107 Chemnitz, Germany. E-mail: stefan.spange@chemie.tu-chemnitz.de; Fax: +49 (0)371-531-21239; Tel: +49 (0)371-531-21230

${ }^{b}$ Institute of Physical Chemistry and Abbe Center of Photonics, Friedrich-Schiller University Jena, Helmholtzweg 4, o7743 Jena, Germany

${ }^{c}$ Leibniz Institute of Photonic Technology (IPHT) Jena e.V., Albert-Einstein-Str. 9, 07745 Jena, Germany

${ }^{d}$ Ivoclar Vivadent AG, Bendererstrasse 2, FL-9494 Schaan, Liechtenstein

$\dagger$ Electronic supplementary information (ESI) available: DFT theoretical calculations, Catalán parameter set, UV/Vis absorption and emission maxima of all compounds, and linear solvation energy relationships. See DOI: 10.1039/c5nj00256g
}

electrostatic effects (dipolarity/polarizability) on the UV/Vis band $\left(\tilde{\nu}_{\max }\right)$ the concept of linear free solvation energy relationship (LSER) is used. ${ }^{6}$

During the last few decades, multiple polarity effects of various environments have been considered in terms of the KamletAbboud-Taft approach (eqn (1)), which has been established as a suitable tool for this purpose.

$$
\tilde{\nu}_{\max }=\tilde{\nu}_{\max , 0}+a \cdot \alpha+b \cdot \beta+s \cdot \pi^{*}
$$

Kamlet, Abboud, and Taft used basically three different polarity parameters which describe the overall solvent polarity: acidity $\alpha$ (hydrogen-bond donor capacity), basicity $\beta$ (hydrogenbond acceptor capacity) and dipolarity/polarizability $\pi^{*}{ }^{6 a, b, 7}$ $\tilde{\nu}_{\text {max }}$ is the longest wavelength UV/Vis maxima of the compound measured in a particular solvent; $\tilde{\nu}_{\max , 0}$ corresponds to a standard process referenced to cyclohexane as a nonpolar medium; $a, b$, and $s$ are solvent-independent correlation coefficients that reflect the contribution of each parameter to the overall solute-solvent interactions.

Unfortunately, the Kamlet-Abboud-Taft concept does not distinguish between dipolarity and polarizability effects both of which are involved in a single $\pi^{*}$-parameter. Therefore, the improved solvent polarity parameter scales of Catalán (eqn (2)) should be used which allow dipolarity and polarizability effects of a solvent to be considered independently of each other. ${ }^{8}$

$$
\tilde{\nu}_{\max }=\tilde{\nu}_{\max , 0}+a \cdot \mathrm{SA}+b \cdot \mathrm{SB}+d \cdot \mathrm{SP}+e \cdot \mathrm{SdP}
$$

$\tilde{\nu}_{\max }$ describes the solvent-dependent physico-chemical properties in a particular solvent and $\tilde{\nu}_{\max , 0}$ the solute 
properties of a reference system, which is defined for the gas phase. The SA (solvent acidity) scale ${ }^{8 a, b, e}$ has been established as a measure of the hydrogen-bond donor (HBD) and electron pair acceptor (EPA) properties, whereas the SB (solvent basicity) scale ${ }^{8 c, e}$ is responsible for the hydrogen-bond acceptor (HBA) and electron pair donor (EPD) properties. The nonspecific interactions polarizability and dipolarity are reflected by the parameters $\mathrm{SP}^{8 d, e}$ and $\mathrm{SdP},{ }^{8 e}$ respectively. $a$, $b, d$, and $e$ are the correlation coefficients characterising the sensitivity of the property $\tilde{\nu}_{\max }$ to different solute-solvent interaction mechanisms.

Fluorosolvatochromism, compared to the comprehensively reported solvatochromism of push-pull substituted chromophores, is still not widely established for measuring environmental effects because only a few probes are known which show a distinct function of $\tilde{\nu}_{\text {max,em }}$ as a function of KamletAbboud-Taft or Catalán solvent parameter. Allegeable solvent effects on the shift of $\tilde{\nu}_{\text {max,em }}$ indicated as fluorosolvatochromism are reported for a few dyes and interpreted in detail concerning specific effects. ${ }^{8 e, 9}$ So far, multiple square correlation analyses of $\tilde{\nu}_{\text {max,em }}$ as a function of various solvent properties are still not established taking into account a numerous set of solvents.

In this work, we present four acetophenone derivatives bearing the heterocycles furan or thiophene in the paraposition to the acetyl unit as a promising class of fluorosolvatochromic compounds. Cross-coupling reactions such as Heck, Stille, Suzuki, Sonogashira, and Negishi type reactions provide an easy route to link carbon atoms of different aryl compounds. Especially by means of the application of the nucleophilicityelectrophilicity principle, those reactions are well manageable. ${ }^{10}$ A nucleophilic (metal) aryl compound reacts with an aryl halide under transition metal catalysis. Palladium has particularly proven to be the transition metal of choice. It bears an extraordinary potential as an activation agent for oxidative addition, insertion, reductive elimination and $\beta$-hydride elimination. ${ }^{11}$

The main focus of the work concentrates on the synthesis, photophysical properties and fluorosolvatochromism ${ }^{12}$ of the acetophenone derivatives. Furan (para electrophilic substituent \left. constant ${\sigma_{\mathrm{p}}}^{+}=-0.39\right)^{13}$ and thiophene $\left(\sigma_{\mathrm{p}}{ }^{+}=-0.43\right)^{13}$ are wellknown electron-donating heterocycles that are widely used to lower the energy band of conjugated materials. These heterocycles are indeed weaker donor substituents as the methoxy group $\left(\sigma_{\mathrm{p}}{ }^{+} \mathrm{OCH}_{3}=-0.78\right),{ }^{13}$ but they offer the option to enhance the length of the chromophoric $\pi$-electron system. This implicates a decrease in the energetic HOMO-LUMO gap and results in a shift towards an even lower-energy absorption range.

\section{Results and discussion}

Synthesis of furanyl- or thiophenyl-substituted acetophenones

In this work, the palladium-catalysed Suzuki and Heck coupling served as a synthesis tool for the $\mathrm{C}-\mathrm{C}$ bond linkage between

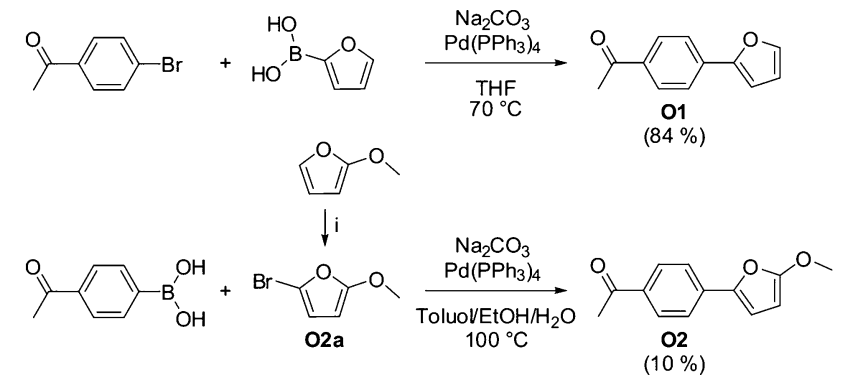

Scheme 1 Synthesis of the functionalized acetophenones $\mathbf{O} 1$ and $\mathbf{O} 2$ using the palladium-catalysed Suzuki coupling. Reaction conditions for (i): $\mathrm{N}$-bromosuccinimide, toluene, cat. $p$-toluenesulfonic acid, $70{ }^{\circ} \mathrm{C}, 4 \mathrm{~h}$.

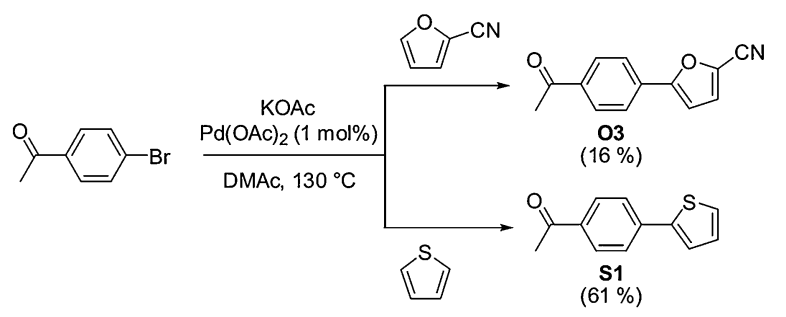

Scheme 2 Synthesis of the functionalized acetophenones O3 and S1 using the palladium-catalysed Heck coupling.

acetophenone and a furan or thiophene moiety. By modifying the furan part with electron donor $(-\mathrm{OMe})$ or acceptor $(-\mathrm{CN})$ moieties it was possible to achieve a structure-property relationships.

In the Suzuki reaction, an aryl halide (in the case of $\mathbf{0 1}$ : 4-bromoacetophenone, 02: 2-bromo-5-methoxyfuran) was reacted with organoboron compounds (01: 2-furanboronic acid, O2: 4-acetylphenylboronic acid) in the presence of base and tetrakis(triphenylphosphine) palladium(0) (Scheme 1).

However, the functionalized acetophenones $\mathbf{O} 3$ and $\mathbf{S 1}{ }^{14}$ were prepared by Heck coupling. Analogous to ref. 14, 2-cyanofuran was converted with the electron-deficient 4-bromoacetophenone to compound $\mathbf{O 3}$ (Scheme 2).

\section{Theoretical calculations}

DFT theoretical studies have been carried out to gain an insight into the electronic structure of acetophenones. All geometries have been optimized using density functional theory with the B3-LYP functional ${ }^{15}$ in combination with the def2-TZVPP basis set. ${ }^{16}$ The calculated HOMO-LUMO energy gaps of compounds 01, O2, O3, and S1 (see ESI, $\uparrow$ Table S1) present values of the HOMO ranging between $4.045 \mathrm{eV}$ for $\mathbf{O} 1$ and $3.737 \mathrm{eV}$ for $\mathbf{O} 2$. A comparison of the furanyl- or thiophenyl-substituted acetophenone derivatives with acetophenones bearing electrondonating groups (e.g. $-\mathrm{OCH}_{3}$ ) shows a significant decrease of the energy gap between HOMO and LUMO. The linear dependency between the calculated HOMO-LUMO energy gaps and the measured UV/Vis absorption maxima $\tilde{\nu}_{\max }$ (Fig. 1) indicates that $\mathrm{UV} / \mathrm{Vis}$ absorption of acetophenones under investigation is mainly justified by a HOMO-LUMO transition. 


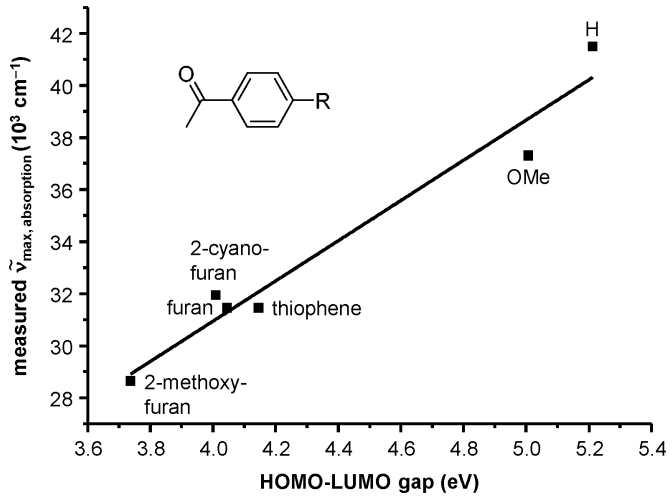

Fig. 1 Experimentally measured UV/Vis absorption maxima of acetophenone derivatives (in DCM) as a function of the theoretically calculated HOMO-LUMO energy differences.

\section{Solvent effects on the UV/Vis absorption spectra}

Optical properties such as UV/Vis absorption and fluorescence of functionalized acetophenones 01, 02, O3, and S1 were investigated both in solution and in the solid state. UV/Vis absorption and emission maxima of the acetophenone derivatives in a variety of solvents are given in the ESI† (Table S3). Fig. 2 exemplarily shows the UV/Vis absorption and emission spectra of the respective compounds recorded in four selected solvents.

All compounds show the shortest wavelength UV/Vis absorption band in $n$-hexane. The compounds $\mathbf{O 1}$ and $\mathbf{S 1}$ exhibit the longest wavelength UV/Vis absorption band in acetone, while O3 shows the strongest bathochromic shift in hexamethylphosphoramide (HMPA). Methoxy-functionalized acetophenone $\mathbf{O} 2$ presents the longest wavelength UV/Vis absorption band in alcohols such as 1-butanol or 1-propanol. These UV/Vis shifts correspond to a positive solvatochromism with a solvatochromic range of $\Delta \tilde{\nu}(\mathbf{O 1})=2140 \mathrm{~cm}^{-1}, \Delta \tilde{\nu}(\mathbf{O 2})=1340 \mathrm{~cm}^{-1}, \Delta \tilde{\nu}(\mathbf{O 3})=$ $2350 \mathrm{~cm}^{-1}$, and $\Delta \tilde{\nu}(\mathbf{S 1})=2050 \mathrm{~cm}^{-1}$. Considering the furan derivatives, it is obvious that the solvatochromic range increases with increasing electron-withdrawing character of the substituent in the 5-position ( $\mathrm{OMe}<\mathrm{H}<\mathrm{CN}$ ).

Furthermore, it is quite evident that an enlargement of the conjugated $\pi$-system causes a bathochromic shift of the absorption wavelength as a consequence. The UV/Vis absorption maxima of the furan- and thiophene-functionalized acetophenones $\mathbf{O 1}$ and $\mathbf{S 1}$ are each detected at $318 \mathrm{~nm}$ in DCM (nonHBA/HBD-solvent). A bathochromic shift of $5900 \mathrm{~cm}^{-1}$ is observed in relation to the UV/Vis absorption maximum of 4-methoxy-acetophenone $\left(\lambda_{\max }=268 \mathrm{~nm}\right)$. A stronger push-pull system is generated by the additional introduction of a methoxy group on the furan ring (O2). In comparison to the unsubstituted heterocyclic compounds $\left(\lambda_{\max }=318 \mathrm{~nm}\right)$, this result causes an enormous bathochromic shift of the UV/Vis absorption maximum at $349 \mathrm{~nm}$, measured in DCM. However, the electron-withdrawing substituent $-\mathrm{CN}$ in $\mathbf{O 3}$ effects a slight hypsochromic shift $\left(\lambda_{\max }=313 \mathrm{~nm}\right.$, in DCM).

Additionally, the UV/Vis spectroscopic behaviour of 01, O2, O3, and S1 was investigated in the solid-state. The UV/Vis
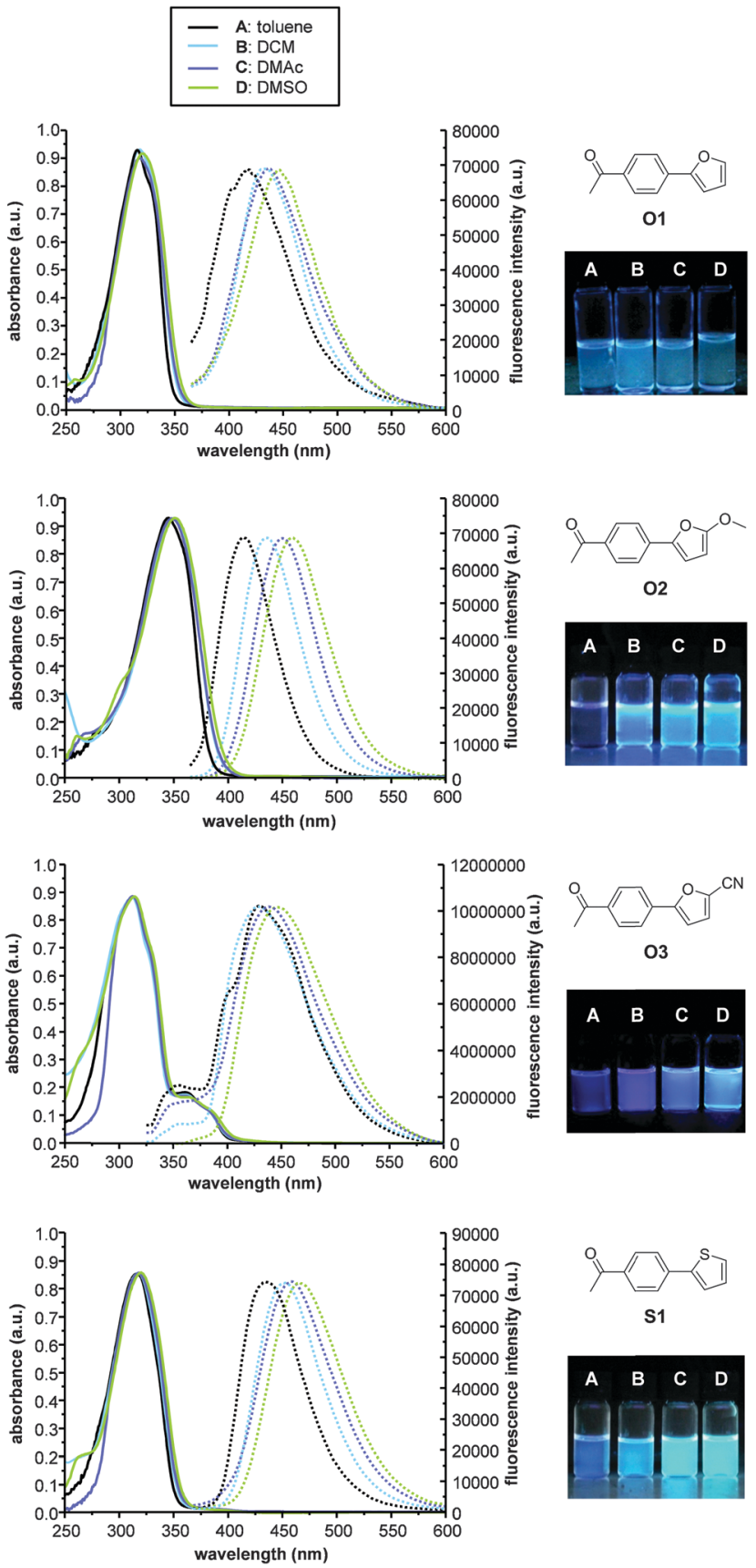

Fig. 2 Normalized UV/Vis absorption and emission spectra of O1, 02, 03, and $\mathbf{S 1}$ measured in toluene (A), DCM (B) DMAC (C), and DMSO (D); $\lambda_{\text {exc }}=$ $310 \mathrm{~nm}\left(\mathbf{O 1}, \mathbf{O 3}\right.$, and S1), $\lambda_{\mathrm{exc}}=350 \mathrm{~nm}(\mathbf{O 2})$.

absorption and emission spectra of the solids are shown in Fig. 3. Generally, UV/Vis absorption bands are non-symmetric and very broad whereby the band broadening of the compounds with substituents in the 5-position of the furan ring (O2 and O3) is significantly more pronounced.

\section{Solvent effects on the UV/Vis emission spectra}

In contrast to the UV/Vis absorption maxima, the UV/Vis emission maxima of the functionalized acetophenones $\mathbf{0 1}$, 


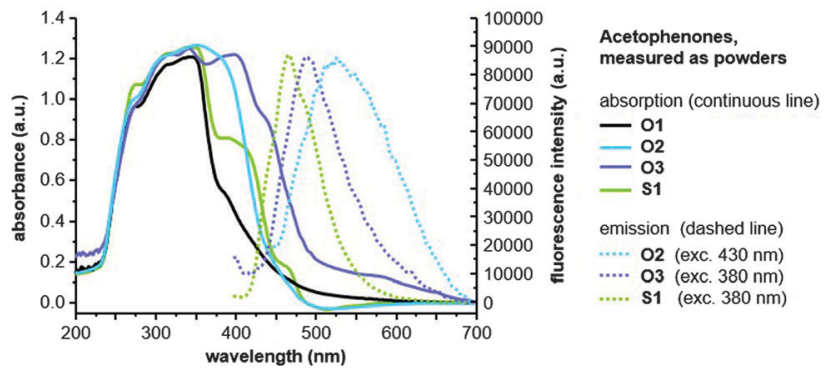

Fig. 3 UV/Vis absorption and emission spectra of $\mathbf{0 1}, \mathbf{0 2}, \mathbf{0 3}$, and $\mathbf{S 1}$ as powders.

$\mathbf{O} 2, \mathbf{O 3}$, and $\mathbf{S 1}$ show a pronounced shift as a function of solvent properties, as illustrated in Fig. 2.

The shortest wavelength UV/Vis emission band was detected in 2-propanol $\left(\lambda_{\max }(\mathbf{O 1})=405 \mathrm{~nm}\right)$, tetrachloromethane $\left(\mathrm{CCl}_{4}\right.$, $\left.\lambda_{\max }(\mathbf{O} 2)=398 \mathrm{~nm}, \lambda_{\max }(\mathbf{O 3})=419 \mathrm{~nm}\right)$ and triethylamine $\left(\lambda_{\max }(\mathbf{S 1})=404 \mathrm{~nm}\right)$. The compounds $\mathbf{O 2}$ and $\mathbf{0 3}$ bearing substituents on the furan ring exhibit the longest wavelength emission band in alcohols $\left(\lambda_{\max }(\mathbf{O} 2)=479 \mathrm{~nm}\right.$ in $\mathrm{MeOH}$, $\lambda_{\text {max }}(\mathbf{O 3})=483 \mathrm{~nm}$ in 2,2,2-trifluoroethanol (TFE)), whereas the furan-/thiophene-functionalized acetophenones show the longest wavelength emission band in the solvents $\gamma$ butyrolactone and $N, N$-dimethylformamide $\left(\mathrm{DMF}, \lambda_{\max }(\mathbf{O 1})=\right.$ $467 \mathrm{~nm}$ ) as well as in dimethyl sulfoxide (DMSO, $\lambda_{\max }(\mathbf{S 1})=$ $466 \mathrm{~nm})$. In this context, the respective UV/Vis shifts correspond to a positive fluorosolvatochromism, but the solvatochromic range of $\Delta \tilde{\nu}(\mathbf{O 1})=3280 \mathrm{~cm}^{-1}, \Delta \tilde{\nu}(\mathbf{O 2})=4260 \mathrm{~cm}^{-1}$, $\Delta \tilde{\nu}(\mathbf{O 3})=3170 \mathrm{~cm}^{-1}$ and $\Delta \tilde{\nu}(\mathbf{S 1})=3290 \mathrm{~cm}^{-1}$ is considerably larger.

The UV/Vis spectroscopic results (absorption and fluorescence) of individual compounds demonstrate that 01, O3 and $\mathbf{S 1}$ absorb at shorter wavelengths compared to $\mathbf{O 2}$, but the fluorescence is observed at similar wavelengths. Thus, an exceptionally large Stokes shift (in DCM: $\Delta \tilde{\nu}(\mathbf{O 1})=8360 \mathrm{~cm}^{-1}$, $\left.\Delta \tilde{\nu}(\mathbf{O 2})=5700 \mathrm{~cm}^{-1}, \Delta \tilde{\nu}(\mathbf{O 3})=8590 \mathrm{~cm}^{-1}, \Delta \tilde{\nu}(\mathbf{S 1})=9280 \mathrm{~cm}^{-1}\right)$ has been obtained (see ESI, $\dagger$ Table S3).

The Stokes shifts of these furanyl- or thiophenyl-substituted acetophenones can be evaluated as disproportionately large compared to other fluorescent dyes, e.g. coumarin derivatives $\left(\Delta \tilde{\nu}\right.$ of $\left.4200-5300 \mathrm{~cm}^{-1}\right) \cdot{ }^{17}$ However, acetophenone bearing a pyrene function in the para-position shows a comparably large Stokes shift in alcohol solutions $\left(\Delta \tilde{\nu} \sim 10000 \mathrm{~cm}^{-1}\right)$ for $\mathbf{S 1} .^{18}$ One of the reasons for the large Stokes shift is certainly that the functionalized acetophenones have a higher dipole moment in the first excited state $S_{1}$ than in the ground state $S_{0}$. Associated with this property a change in the dipole moment in strength and orientation is induced. The solvent molecules respond with a reorganization of the solvent shell around the excited acetophenone molecules. Hence, the state $S_{1}$ of the acetophenone molecule is better solvated by polar solvents and as a result its energetical stabilization is improved. The electronic state after emission $S_{0, \text { emission }}$ is energetically unfavourably solvated due to the shorter timescale of photon emission in comparison to solvent molecule reorganization. After relaxation of the solvent

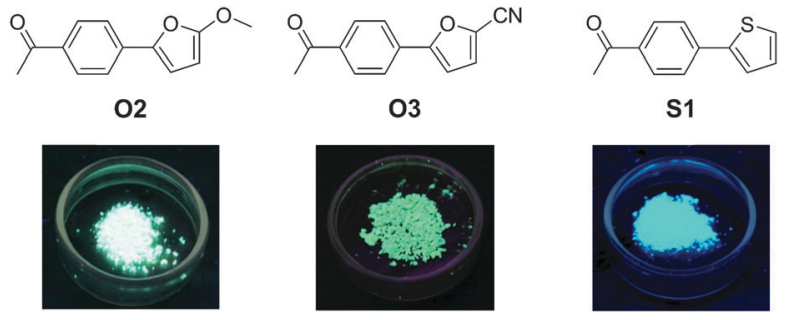

Fig. 4 Photographs of the solid-state fluorescence of acetophenones O2, O3, and S1, excited at $380 \mathrm{~nm}(\mathbf{O 3}, \mathbf{S 1})$ and $430 \mathrm{~nm}$ (O2).

molecules the equilibrium state $S_{0}$ is reached. The difference in the energies of $S_{0, \text { emission }}$ and $S_{0}$ adds to a strongly red-shifted fluorescence of the acetophenone systems.

Methoxyfuran-, cyanofuran-, and thiophene-functionalized acetophenones $(\mathbf{O} 2, \mathbf{O 3}$, and $\mathbf{S 1})$ also demonstrate a pronounced solid-state fluorescence (Fig. 4). However, compound 01 shows no fluorescence in the solid-state.

These observations indicate that the substitution in the 5-position of the furan ring or the exchange of oxygen with sulphur plays an important role in tuning the photochemical properties of these types of acetophenones. Fluorescence spectroscopic measurements of the functionalized acetophenones $\mathbf{O} 2$, O3, and $\mathbf{S 1}$ in the solid-state are also shown in Fig. 3. The UV/Vis emission maxima of methoxyfuran-substituted acetophenone (O2) is located at $524 \mathrm{~nm}$ which is red-shifted by $\Delta \tilde{\nu}=$ $1500 \mathrm{~cm}^{-1}$ compared to its cyanofuran-substituted analogue O3. Compound $\mathbf{S 1}$ is fluorescent with a UV/Vis emission band at $465 \mathrm{~nm}$. It is evident that the solid-state fluorescence of methoxyfuran-functionalized acetophenone (O2) is strongly red-shifted by $\Delta \tilde{\nu}=1800 \mathrm{~cm}^{-1}$ in comparison to the lowestenergy emission band measured in polar solvents (Fig. 2 and 3). The UV/Vis emission maxima of the solids $\mathbf{0 3}$ and $\mathbf{S 1}$ are similar to those in solution (polar solvents, e.g. $\lambda_{\max }(\mathbf{O 3})=$ $483 \mathrm{~nm}$ in TFE, $\lambda_{\max }(\mathbf{S 1})=466 \mathrm{~nm}$ in DMSO).

\section{Determination of excited-state lifetimes}

The fluorescence decay profiles of the furanyl- or thiophenylfunctionalized acetophenones 01, O2, O3, and S1 were measured by time-correlated single photon counting (TCSPC, Fig. 5). The emission lifetimes are in the order of 0.2 to $2 \mathrm{~ns}$ (Table 1) and support the assignment of the emission to be due to fluorescence. For $\mathbf{O} 2$ and $\mathbf{O 3}$ the fluorescence decay-curves were found to be bi-exponential, while for the other substances exponential decays were observed. There is no unique relationship between solvent polarity and emission lifetime. Nevertheless, it is noted that for $\mathbf{O 1}$ and $\mathbf{O 2}$ the lifetime in DCM is longer than in ethanol (EtOH), while the opposite holds true for $\mathbf{O 3}$ and S1. This points to significant differences in the electronic structures of the excited states and the impact of specific solute-solvent interactions, e.g. hydrogen-bonding ability.

\section{Linear solvation energy correlation analyses}

The influence of the solvent behaviour on the UV/Vis shift of the absorption and emission maxima has been determined by 

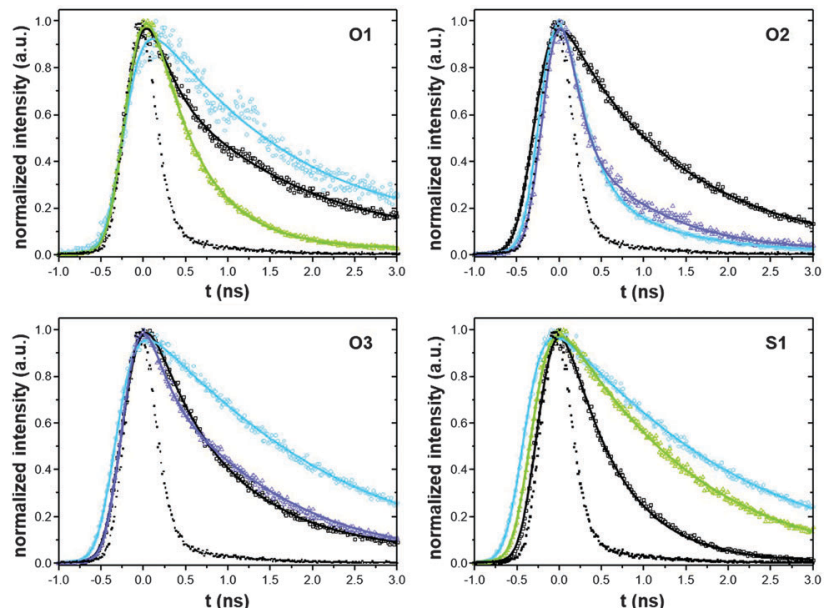

Fig. 5 Emission decay curves (hollow symbols) obtained via TCSPC $\left(\lambda_{\text {exc }}\right.$ $=350 \mathrm{~nm}$ ) for O1, O2, O3, and S1 in different solvents: DCM (-), EtOH (-), DMF (-) and $\mathrm{CCl}_{4}(-)$. The solid lines represent the respective fit curves (see the Experimental section). The solid symbols represent the instrumental response function.

Table 1 Emission lifetimes and fit parameters of $\mathbf{0 1}$, O2, O3, and $\mathbf{S 1}$ in selected solvents

\begin{tabular}{lllll}
\hline & \multicolumn{4}{c}{$\tau(\mathrm{ns})$} \\
\cline { 2 - 5 } Compd & DCM & EtOH & $\mathrm{CCl}_{4}$ & \multicolumn{1}{c}{ DMF } \\
\hline O1 & 1.4 & 0.5 & - & $0.2(65 \%)$ \\
& & & $1.5(35 \%)$ \\
O2 & $0.4(49 \%)$ & $0.2(93 \%)$ & $<0.2(88 \%)$ & - \\
& $1.4(51 \%)$ & $1.2(7 \%)$ & $0.9(12 \%)$ & \\
O3 & $0.6(86 \%)$ & 1.9 & $0.2(65 \%)$ & - \\
& $2.0(14 \%)$ & & $1.3(35 \%)$ & \multirow{2}{*}{1.3} \\
S1 & 0.5 & 1.8 & - & \\
\hline
\end{tabular}

means of multiple linear regression analyses using the solvent polarity parameter set of Catalán. ${ }^{19}$ The solvatochromism of the furanyl- or thiophenyl-functionalized acetophenones was studied in 26 solvents with dipolarities/polarizabilities and hydrogen-bonding capacities.

However, the HBD/HBA abilities of the environment have only a marginal influence upon the UV/Vis absorption maxima of the solvatochromic probe.

The results of multiple linear regression analysis of the solvent dependent emission band of substituted acetophenones $\mathbf{0 1}, \mathbf{O 2}$, $\mathbf{O 3}$, and $\mathbf{S 1}$, which are qualitatively the best according to the solvent scale of Catalán, are compiled in Table 2. The correlation coefficients $r$ are greater than 0.9 (with the exception of O1) for the
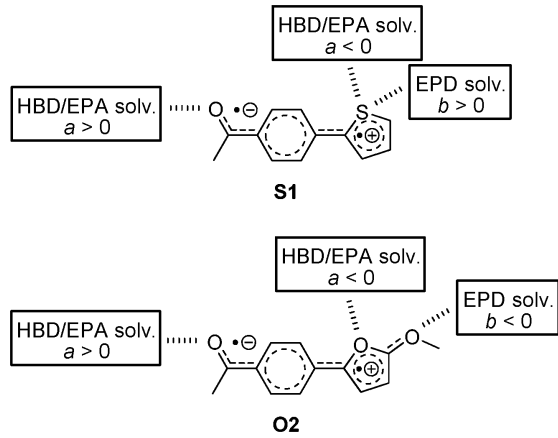

Fig. 6 Possible hydrogen-bond (HB) and electron pair (EP) interactions between solute and solvents, and their influence on the emission as demonstrated for the compounds $\mathbf{S 1}$ and $\mathbf{0 2}$, both are shown in an assumed excited state.

LSE relationships. These results indicate a very high validity of the obtained multiparameter equations and allow significant conclusions on the fluorosolvatochromic behaviour.

In this context, the solvent independent correlation coefficient $a$ provides information about solvent interactions with the hydrogen-bond acceptor/electron pair donor fragment (i.e. lone electron pair of the partially negatively charged oxygen from the keto group) of the respective dye. However, the coefficient $b$ describes specific solvation of the dye by the hydrogen-bond donor/electron pair acceptor group using appropriate HBA/ EPD-solvents. Fig. 6 shows, for instance, the assumed electron distribution of compounds $\mathbf{S 1}$ and $\mathbf{O 2}$ in the excited state with possible specific solute-solvent interactions which can have an influence on the fluorosolvatochromic behaviour.

A positive sign indicates that the push-pull system is decreased by the solute-solvent interactions and this results in a hypsochromic shift. Consequently, a negative sign is associated with a bathochromic shift. The appearance of the latter is associated with an enhancement of the push-pull character of the dye.

Surprisingly, the calculated LSER of the solvent dependent emissions of furan-/thiophene-functionalized acetophenones $(\mathbf{O 1}, \mathbf{S 1})$ show that the solvent independent correlation coefficients $a$ and $b$ are positive. In this case protic solvents, which can act as hydrogen-bond donors, mainly interact with the negatively charged oxygen atom of the keto group $(a>0)$.

As a result, the $(+\mathrm{M})$-effect of the $\mathrm{O}^{-}$group is decreased and also the push-pull character of the aromatic system is weakened.

A weakening of the push-pull system can also be observed by interactions of EPD-solvents with acetophenones $\mathbf{0 1}$ and S1.

Table 2 Solvent-independent correlation coefficients $a, b, d$ and e of the Catalán parameters SA, SB, SP and SdP, solute property of the reference system $\tilde{\nu}_{\max , 0}$ gas phase, number of solvents $(n)$, correlation coefficient $(r)$, standard deviation (sd), and significance $(f)$ of the fluorosolvatochromism of the acetophenone-functionalized compounds $\mathbf{0 1}, \mathbf{0 2}, \mathbf{0 3}$, and S1

\begin{tabular}{lcccccccccc}
\hline Entry & Compound & $\tilde{\nu}_{\text {max }, 0}\left(10^{3} \mathrm{~cm}^{-1}\right)$ & $a$ & $b$ & $d$ & $e$ & $n$ & $r$ & sd \\
\hline 2 & O1 & 26.926 & 1.198 & 1.936 & -4.204 & -2.847 & 23 & 0.841 & 0.591 & $\leq 1.175 \times 10^{-4}$ \\
3 & $\mathbf{O}$ & 24.787 & -2.768 & -0.993 & - & -1.887 & 25 & 0.960 & 0.351 & $\leq 9.269 \times 10^{-12}$ \\
4a & $\mathbf{O 3}$ & 23.111 & -2.808 & - & - & - & 31 & 0.920 & 0.360 & $\leq 2.353 \times 10^{-13}$ \\
4b & $\mathbf{O 3}$ & 22.984 & -2.619 & - & - & - & 12 & 0.870 & 0.258 & $\leq 9.735 \times 10^{-6}$ \\
5 & S1 & 25.466 & 1.958 & 1.917 & -3.322 & -2.202 & 24 & 0.929 & 0.349 & $\leq 6.051 \times 10^{-8}$
\end{tabular}


Thereby, EPD-solvents interact predominantly with the oxygen or sulphur of the heterocyclic ring due to its positive charge located in the excited state $(b>0)$. The strongest effect of the fluorosolvatochromic behaviour of $\mathbf{O 1}$ and $\mathbf{S 1}$ is exerted by the polarizability (SP) and the dipolarity (SdP) of the solvents. The respective coefficients $d$ and $e$ have a negative value and thus indicate positive fluorosolvatochromism. Here, a bathochromic shift of the emission maxima is observed with increasing polarizability and dipolarity of the solvent. Considering the emission spectra of the functionalized acetophenones O1, O2, O3, and S1 (Fig. 2) reveal that the compounds $\mathbf{O 1}$ and $\mathbf{S 1}$ show a smaller difference between the emission maxima of non-polar solvents (i.e. toluene) and those of polar solvents (i.e. DMSO). One possible explanation for these observation could be that the terms of the nonspecific interactions ( $d$ and $e$ ) exhibit negative values, but the coefficients $a$ and $b$ are positive. The sum of all solvent interactions results in a bathochromic shift, but it is not as large as the shift of acetophenone $\mathbf{0 2}$, as can be seen in the following interpretation.

In contrast to $\mathbf{O} \mathbf{1}$ and $\mathbf{S 1}$, the functionalized acetophenone O2 shows a negative sign of parameter $a$, which indicates that the interaction of $\mathrm{HBD} / \mathrm{EPA}$-solvents with the lone electron pairs of oxygen atoms of the furan ring is significantly stronger than those with oxygen of the keto group $(a<0)$. Therefore, electron density is donated by the furan ring to the respective solvent molecules. This effect induces a reinforcement of the push-pull system and finally a bathochromic shift is caused. The correlation coefficient $b$ of $\mathbf{O 2}$ obtained from the LSE correlation analyses also illustrates a bathochromic shift, but it is less pronounced. Furthermore, the correlation of $\mathbf{O} 2$ shows that the absolute $e$ value has a lower influence than the coefficient $a$. This result demonstrates that the UV/Vis emission spectrum is weakly dependent on the change in the dipolarity of the chromophore environments rather than on the capacity of the solvent to act as a hydrogen-bond donor. Surprisingly, $\mathbf{O 2}$ shows no influence with regard to the polarizability (coefficient $d$ ) of the solvent. Fig. 7 exemplarily shows the linear relationship

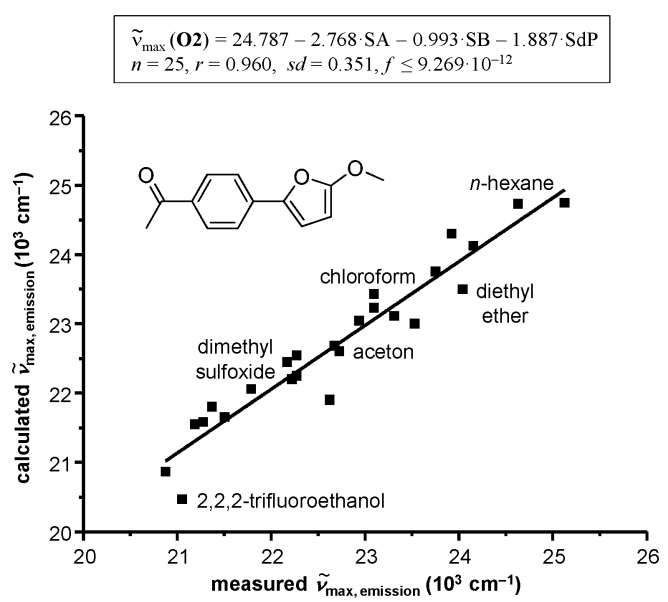

Fig. 7 Linear solvation energy relationship for the fluorescence of $\mathbf{O} 2$ in 25 various solvents. Plots of measured vs. calculated emission maxima, according to the Catalán equation are shown.

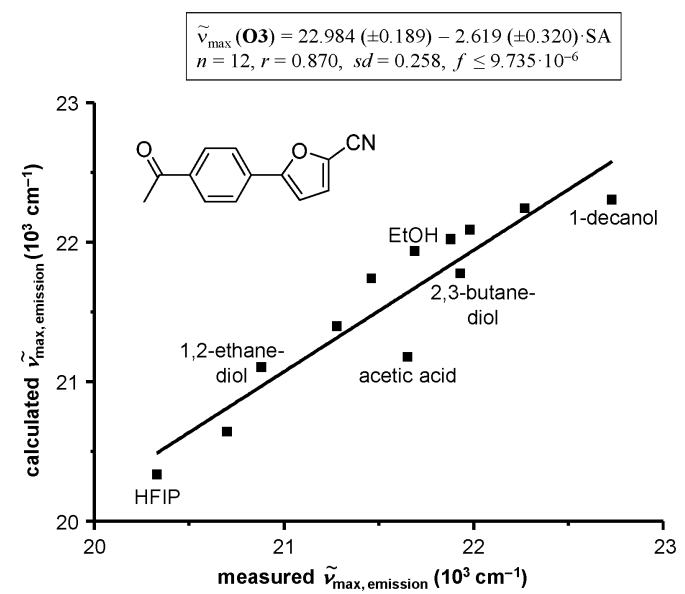

Fig. 8 Linear solvation energy relationship between calculated and measured fluorescence $\tilde{\nu}_{\text {max,em }}$ for $\mathbf{0 3}$ in 12 solvents with hydrogen-bond donating ability.

between the measured and calculated wavenumbers for the solvent dependent UV/Vis emission band of acetophenone $\mathbf{O} 2$.

In comparison to the methoxyfuran-functionalized acetophenone, the result of multiple linear regression analysis of a CN analogue $\mathbf{O 3}$ shows that its SA sensitivity is of highest significance as shown by the equation in Fig. 8. Therefore, the UV/Vis emission maxima of $\mathbf{O 3}$ were described using the single-parameter equation (Table 2, entry 4a) and show an excellent correlation.

Fig. 8 displays the plot of the calculated UV/Vis maxima for the emission as a function of the corresponding experimental values for only classic HBD-solvents.

\section{Conclusions}

In this work, four furanyl- or thiophenyl-functionalized acetophenones have been produced by means of palladium-catalysed cross-coupling reactions such as Heck or Suzuki coupling. Optical properties (UV/Vis absorption and emission) were been studied both in solution and in the solid-state. The represented results show that the UV/Vis absorption spectra of the substituted acetophenones $\mathbf{0 1}, \mathbf{O 2}, \mathbf{O 3}$, and $\mathbf{S 1}$ do not noticeably shift as the solvent is varied. In contrast, the emission spectra exhibit a strong solvent-dependence.

Due to the dependence of the emission maxima on the nature of the solvent, the fluorosolvatochromic behaviour of the furanyl- or thiophenyl-substituted acetophenones was studied in solvents of different acidity, basicity and polarity. The results of LSER correlations using the Catalán parameter set suggest that the influence of solvent dipolarity/polarizability on the long wavelength emission maximum for $\mathbf{O 1}$ and $\mathbf{S 1}$ is predominant. Furthermore, the influence on the fluorosolvatochromic behaviour of $\mathbf{O} 2$ is reflected by negative signs of $a, b$ and $e$ coefficients, whereby the strongest is exerted by the acidity of the solvent. A single-parameter equation according to Catalán was established for compound O3. Accordingly, the fluorosolvatochromic 
dye $\mathbf{O 3}$ can be used as a HBD/EPA-sensitive probe for several environments (solvent and solid-state).

Acetophenones $\mathbf{O 2}, \mathbf{O 3}$, and $\mathbf{S 1}$ fluoresce in solution as well as in the solid-state. However, the solid-state fluorescence of $\mathbf{O 1}$ is not observed.

\section{Experimental section}

\section{Materials}

Organic solvents were dried according to standard methods in an argon atmosphere and freshly distilled prior to use. All commercial reagents were used without further purification. They were purchased from the following suppliers: Acros: 4-bromoacetophenone (98\%), 2-cyanofuran (99\%), thiophene ( $>99 \%)$, potassium acetate (97\%), and $p$-toluenesulfonic acid (99\%); Alfa Aesar: 2-methoxyfuran (97\%); ChemPur: tetrakis(triphenylphosphine) palladium(0) (99\%); Fluka: palladium(II) acetate (47\% Pd); Fluorochem: 4-acetylphenylboronic acid (98\%) and furan-2-boronic acid (98\%); Merck: $\mathrm{N}$-bromosuccinimide (99\%).

\section{Instrumentation}

The melting points were measured on a hot-stage microscope using a digital temperature measuring system from Wagner \& Munz and are uncorrected. Differential scanning calorimetry (DSC) measurements were accomplished using a Mettler Toledo, type DSC 30. Elemental analyses were determined using a Vario-EL analyser. Attenuated total reflection infrared (ATR IR) spectra were recorded using a Golden Gate ATR accessory on a Perkin-Elmer Fourier transform 1000 spectrometer at room temperature in the wavenumber range 400 to $4000 \mathrm{~cm}^{-1} \cdot{ }^{1} \mathrm{H} \mathrm{NMR}(250 \mathrm{MHz})$ and ${ }^{13} \mathrm{C}$ NMR (69.9 MHz) spectroscopy were carried out on a Bruker Avance 250 NMR spectrometer. The residue signals of the solvents were used as internal standards. High-resolution mass spectra were recorded on a Bruker Daltonik microTOF-QII spectrometer. UV/Vis absorption spectra were obtained on a MCS 400 diode array UV/Vis spectrometer from Carl Zeiss, Jena, connected via glass-fibre optics. Fluorescence spectra were recorded on a Fluoromax $^{\circledR}-4$ Horiba Jobin Yvon spectrometer. Excitation and emission slits were set to $5 \mathrm{~nm}$. The excitation wavelength was varied between $310 \mathrm{~nm}$ (O1, O3, and S1) and $350 \mathrm{~nm}(\mathbf{O 2})$. Correlation analysis: multiple regression analysis was performed using the Origin Pro 9.0G statistics program. The emission decay curves were obtained via time-correlated-single-photon-counting. After excitation with a frequency-doubled Ti-sapphire laser adjusted to $700 \mathrm{~nm}$ (Tsunami, Newport Spectra-Physics GmbH, pulse-to-pulse repetition rate $800 \mathrm{kHz}$ after passing a pulse selector, model 3980, Newport Spectra-Physics $\mathrm{GmbH}$ ), i.e. at $\lambda_{\text {exc }}=350 \mathrm{~nm}$, the luminescence of the sample was collected in a $90^{\circ}$-geometry and detected using a Becker \& Hickl PMC-100-4 photon-counting module. Two long-pass filters (355 $\mathrm{nm}$ and $395 \mathrm{~nm}$ ) are inserted into the detection beam path. The instrumental response function (IRF) is measured using a scattering sample (P25 nanoparticles in water). The measured curves are fitted mono- or bi-exponentially including the convolution with the IRF trace using a home-written algorithm in Scilab. ${ }^{20}$

\section{Methods}

All geometries have been optimized using density functional theory with the B3-LYP functional ${ }^{15}$ in combination with the def2-TZVPP basis set. ${ }^{16}$ The geometries have been converged to a residual gradient norm of $10^{-3}$ a.u. or better. The energy was converged to at least $10^{-6}$ a.u., and fine quadrature grids have been used to calculate the exchange and correlation potential (size 5 ). ${ }^{21}$ All minima have been characterized by normal-mode analysis. All calculations have been performed using the TURBOMOLE program package (for a current version, see http:// www.turbomole.de). ${ }^{22}$

\section{Syntheses}

4-(Furan-2-yl)acetophenone (O1). 4-Bromoacetophenone (3.23 g, $16.25 \mathrm{mmol})$, furan-2-boronic acid (2.00 g, $17.87 \mathrm{mmol})$, $\mathrm{Pd}\left(\mathrm{PPh}_{3}\right)_{4}(1.88 \mathrm{~g}, 16.25 \mathrm{mmol})$ and $65 \mathrm{~mL}$ of aqueous $\mathrm{Na}_{2} \mathrm{CO}_{3}$ solution $\left(1.0 \mathrm{~mol} \mathrm{~L}^{-1}\right)$ were mixed in $120 \mathrm{~mL}$ of THF (degassed with argon). The reaction mixture was refluxed under argon for $24 \mathrm{~h}$. To this solution $200 \mathrm{~mL}$ of $\mathrm{HCl}\left(0.1 \mathrm{~mol} \mathrm{~L}^{-1}\right)$ was added and the aqueous phase was extracted with DCM $(2 \times 100 \mathrm{~mL})$. The organic phase was washed with distilled water $(3 \times 100 \mathrm{~mL})$, dried over $\mathrm{MgSO}_{4}$, filtered and concentrated. The crude product was purified by column chromatography on silica gel (eluent: DCM) to obtain $01(2.54 \mathrm{~g}, 84 \%)$ as a yellow to orangecoloured solid.

Mp: $96-98{ }^{\circ} \mathrm{C}$. DSC $\left(25-350{ }^{\circ} \mathrm{C}, 10 \mathrm{~K} \mathrm{~min}^{-1}, \mathrm{~N}_{2}\right): 100{ }^{\circ} \mathrm{C}$ (endo), $230{ }^{\circ} \mathrm{C}$ (endo). Found: $\mathrm{C}, 77.2 ; \mathrm{H}, 5.4$. Calc. for $\mathrm{C}_{12} \mathrm{H}_{10} \mathrm{O}_{2}$ : $\mathrm{C}, 77.4 ; \mathrm{H}, 5.4 \% . \nu_{\max } / \mathrm{cm}^{-1} 3105(\mathrm{ArCH}), 1666(\mathrm{C}=\mathrm{O}), 1602$ $(\mathrm{C}=\mathrm{C}), 1560$ ( $\mathrm{C}=\mathrm{C}$; furan), 1263 (def vib; 1,4-disubstituted benzene), 839 (def vib; 1,4-disubstituted benzene). $\delta_{\mathrm{H}}(250 \mathrm{MHz}$; $\left.\mathrm{CDCl}_{3} ; \mathrm{Me}_{4} \mathrm{Si}\right) 2.61(3 \mathrm{H}, \mathrm{s}, \mathrm{Me}), 6.52(1 \mathrm{H}, \mathrm{dd}, J 3.4 \mathrm{~Hz}, J 1.7 \mathrm{~Hz}$, fuH), $6.81(1 \mathrm{H}, \mathrm{d}, J 3.4 \mathrm{~Hz}$, fuH $), 7.54(1 \mathrm{H}, \mathrm{d}, J 1.7 \mathrm{~Hz}, \mathrm{fuH}), 7.75$ ( $2 \mathrm{H}, \mathrm{d}, J 8.4 \mathrm{~Hz}, \mathrm{ArH}), 7.98(2 \mathrm{H}, \mathrm{d}, J 8.4 \mathrm{~Hz}, \mathrm{ArH}) . \delta_{\mathrm{C}}(62.9 \mathrm{MHz}$, $\mathrm{CDCl}_{3}, \mathrm{Me}_{4} \mathrm{Si}$ ) 26.7, 107.6, 112.3, 123.7, 129.1, 135.0, 135.7, 143.4, 153.0, 197.6.

2-Bromo-5-methoxyfuran (O2a). A mixture of 2-methoxyfuran $(1.50 \mathrm{~g}, 15.29 \mathrm{mmol}), N$-bromosuccinimide $(2.72 \mathrm{~g}$, $15.29 \mathrm{mmol}$ ) and anhydrous $p$-toluenesulfonic acid (8.0 mg) in toluene $(75 \mathrm{~mL})$ was heated for $4 \mathrm{~h}$ at $70{ }^{\circ} \mathrm{C}$. After cooling to room temperature the precipitated succinimide was filtered off. The filtrate was used without further purification for the synthesis of 4-(5-methoxyfuran-2-yl)acetophenone (O2).

4-(5-Methoxyfuran-2-yl)acetophenone (O2). To the solution of the crude product $\mathbf{O 2 a} 20 \mathrm{~mL}$ of ethanol and $20 \mathrm{~mL}$ of distilled water (degassed with argon) were added. Subsequently, 4-acetylphenylboronic acid (2.46 g, $15.00 \mathrm{mmol}), \mathrm{Na}_{2} \mathrm{CO}_{3}$ (1.75 g, $16.50 \mathrm{mmol})$ and $\mathrm{Pd}\left(\mathrm{PPh}_{3}\right)_{4}(1.39 \mathrm{~g}, 1.20 \mathrm{mmol})$ were added. The dark brown reaction mixture was refluxed under argon for about $72 \mathrm{~h}$. After cooling to room temperature $200 \mathrm{~mL}$ of aqueous $\mathrm{HCl}\left(0.1 \mathrm{~mol} \mathrm{~L}^{-1}\right)$ was added to this solution and the aqueous phase was extracted with dichloromethane $(2 \times 100 \mathrm{~mL})$. The organic phase was washed with distilled water $(1 \times 100 \mathrm{~mL})$, dried over $\mathrm{MgSO}_{4}$, filtered and the filtrate was evaporated. The resulting 
crude product was purified by column chromatography using DCM as an eluent to yield $\mathbf{O} 2(0.33 \mathrm{~g}, 10 \%)$ as an orangecoloured solid.

Mp: 88-92 ${ }^{\circ} \mathrm{C}$. DSC $\left(25-350{ }^{\circ} \mathrm{C}, 10 \mathrm{~K} \mathrm{~min}^{-1}, \mathrm{~N}_{2}\right): 92{ }^{\circ} \mathrm{C}($ endo $)$, $181{ }^{\circ} \mathrm{C}$ (exo). $\nu_{\max } / \mathrm{cm}^{-1} 3004(\mathrm{ArCH}), 2838\left(-\mathrm{OCH}_{3}\right), 1667$ $(\mathrm{C}=\mathrm{O}), 1569(\mathrm{C}=\mathrm{C}), 1551(\mathrm{C}=\mathrm{C}$; furan), 1250 (def vib; 1,4disubstituted benzene), 959 ( $\mathrm{CH}$ def vib; 2,5-disubstituted furan), 816 (def vib; 1,4-disubstituted benzene). $\delta_{\mathrm{H}}(250 \mathrm{MHz}$; $\left.\mathrm{CDCl}_{3} ; \mathrm{Me}_{4} \mathrm{Si}\right) 2.59$ (3 H, s, Me), 3.93 (3 H, s, Me), $5.31(1 \mathrm{H}, \mathrm{d}$, $J 3.5 \mathrm{~Hz}, \mathrm{fuH}), 6.71(1 \mathrm{H}, \mathrm{d}, J 3.5 \mathrm{~Hz}, \mathrm{fuH}), 7.60(2 \mathrm{H}, \mathrm{d}, J 8.8 \mathrm{~Hz}$, $\mathrm{ArH}), 7.94(2 \mathrm{H}, \mathrm{d}, J 8.8 \mathrm{~Hz}, \mathrm{ArH}) . \delta_{\mathrm{C}}\left(62.9 \mathrm{MHz}, \mathrm{CDCl}_{3}, \mathrm{Me}_{4} \mathrm{Si}\right)$ 26.5, 57.9, 82.5, 109.5, 122.0, 129.0, 134.5, 134.9, 143.0, 162.4, 197.3. HRMS (ESI-TOF): calc. for $\mathrm{C}_{13} \mathrm{H}_{13} \mathrm{O}_{3}: \mathrm{m} / \mathrm{z}$ 217.0859, found: $m / z 217.0864$.

4-(Thiophen-2-yl)acetophenone (S1). Compound S1 was prepared according to the literature. ${ }^{14}$ Lemon-coloured solid. $61 \%$ yield. Mp: $116-119{ }^{\circ} \mathrm{C}$. DSC $\left(25-350{ }^{\circ} \mathrm{C}, 10 \mathrm{~K} \mathrm{~min}^{-1}, \mathrm{~N}_{2}\right): 120{ }^{\circ} \mathrm{C}$ (endo), $255^{\circ} \mathrm{C}$ (endo). $\nu_{\max } / \mathrm{cm}^{-1} 3072(\mathrm{ArCH}), 1675(\mathrm{C}=\mathrm{O}), 1598$ $(\mathrm{C}=\mathrm{C}), 1559$ ( $\mathrm{C}=\mathrm{C}$; thiophene), 1258 (def vib; 1,4-disubstituted benzene), 822 (def vib; 1,4-disubstituted benzene), 714 (def vib; monosubstituted thiophene). $\delta_{\mathrm{H}}\left(250 \mathrm{MHz} ; \mathrm{CDCl}_{3} ; \mathrm{Me}_{4} \mathrm{Si}\right) 2.61$ (3 H, s, Me), 7.12 (1 H, dd, J $4.9 \mathrm{~Hz}, J 3.0 \mathrm{~Hz}$, thH), $7.36(1 \mathrm{H}, \mathrm{d}$, $J 4.9 \mathrm{~Hz}$, thH $), 7.43(1 \mathrm{H}, \mathrm{d}, J 3.0 \mathrm{~Hz}$, thH $), 7.69(2 \mathrm{H}, \mathrm{d}, J 8.4 \mathrm{~Hz}$, $\mathrm{ArH}), 7.97(2 \mathrm{H}, \mathrm{d}, J 8.4 \mathrm{~Hz}, \mathrm{ArH}) . \delta_{\mathrm{C}}\left(62.9 \mathrm{MHz}, \mathrm{CDCl}_{3}, \mathrm{Me}_{4} \mathrm{Si}\right)$ 26.7, 124.7, 125.8, 126.6, 128.5, 129.3, 135.9, 138.9, 143.1, 197.5. HRMS (ESI-TOF): calc. for $\mathrm{C}_{12} \mathrm{H}_{11} \mathrm{OS}: \mathrm{m} / z$ 203.0525, found: $\mathrm{m} / \mathrm{z}$ 203.0532 $[\mathrm{M}+\mathrm{H}]^{+}$.

2-(4-Acetylphenyl)furan-5-carbonitrile (O3). Compound $\mathrm{O3}$ was synthesized in analogy to the aryl-substituted thiophene derivatives, which were presented in the studies of Roger et al. ${ }^{14}$ 4-Bromoacetophenone $(1.00 \mathrm{~g}, 5.02 \mathrm{mmol})$ was dissolved in $\mathrm{N}, \mathrm{N}$-dimethylacetamide $(15 \mathrm{~mL})$, and 2-cyanofuran $(0.92 \mathrm{~g}$, $9.90 \mathrm{mmol}$ ), potassium acetate (0.99 g, $10.05 \mathrm{mmol})$, and $\mathrm{Pd}(\mathrm{OAc})_{2}(12.40 \mathrm{mg}, 0.06 \mathrm{mmol})$ were added to this solution. The reaction mixture was heated for $46 \mathrm{~h}$ at $150{ }^{\circ} \mathrm{C}$. The resulting solution was added to DCM, the insoluble part was filtered off and the filtrate concentrated. The crude product was purified by column chromatography on silica gel (eluent: DCM/hexane: 4/1) to obtain $\mathrm{O3}(0.172 \mathrm{~g}, 16 \%)$ as an orange-coloured solid.

Mp: $94-108{ }^{\circ} \mathrm{C}$. DSC $\left(25-350{ }^{\circ} \mathrm{C}, 10 \mathrm{~K} \mathrm{~min}^{-1}, \mathrm{~N}_{2}\right): 104{ }^{\circ} \mathrm{C}$ (endo), $230{ }^{\circ} \mathrm{C}$ (endo). $\nu_{\max } / \mathrm{cm}^{-1} 3135(\mathrm{ArCH}), 2226(\mathrm{C} \equiv \mathrm{N})$, $1677(\mathrm{C}=\mathrm{O}), 1609(\mathrm{C}=\mathrm{C}), 1562(\mathrm{C}=\mathrm{C}$; furan), 1268 (def vib; 1,4-disubstituted benzene), 961 ( $\mathrm{CH}$ def vib; 2,5-disubstituted furan), 814 (def vib; 1,4-disubstituted benzene). $\delta_{\mathrm{H}}(250 \mathrm{MHz}$; $\left.\mathrm{CDCl}_{3} ; \mathrm{Me}_{4} \mathrm{Si}\right) 2.64(3 \mathrm{H}, \mathrm{s}, \mathrm{Me}), 6.87$ (1 H, d, J 3.6 Hz, fuH ), 7.21 (1 H, d, J $3.6 \mathrm{~Hz}, \mathrm{fuH}), 7.81(2 \mathrm{H}, \mathrm{d}, J 8.2 \mathrm{~Hz}, \mathrm{ArH}), 8.03(2 \mathrm{H}, \mathrm{d}$, $J 8.2 \mathrm{~Hz}, \mathrm{ArH}) . \delta_{\mathrm{C}}\left(62.9 \mathrm{MHz}, \mathrm{CDCl}_{3}, \mathrm{Me}_{4} \mathrm{Si}\right) 26.8,108.1,111.7$, 124.1, 124.9, 126.2, 129.3, 132.6, 137.4, 157.3, 197.7. HRMS (ESI-TOF): calc. for $\mathrm{C}_{13} \mathrm{H}_{10} \mathrm{NO}_{2}: \mathrm{m} / \mathrm{z}$ 212.0706, found: $\mathrm{m} / \mathrm{z}$ 212.0714 [M+ H $]^{+}$.

\section{Acknowledgements}

Financial support from the Fonds der Chemischen Industrie is gratefully acknowledged. We also thank K. Müller for elemental analyses and Dr. R. Buschbeck and B. Kempe (TU Chemnitz, Prof. Dr. H. Lang group) for mass-spectrometry measurements.

\section{Notes and references}

1 (a) A. Kraft, A. C. Grimsdale and A. B. Holmes, Angew. Chem., Int. Ed., 1998, 37, 402-428; (b) U. Mitschke and P. Bäuerle, J. Mater. Chem., 2000, 10, 1471-1507; (c) C. J. Tonzola, M. M. Alam, W. Kaminsky and S. A. Jenekhe, J. Am. Chem. Soc., 2003, 125, 13548-13558; (d) H.-C. Yeh, L.-H. Chan, W. Wu and C.-T. Chen, J. Mater. Chem., 2004, 14, 1293-1298; (e) C.-T. Chen, Chem. Mater., 2004, 16, 4389-4400; $(f)$ C.-L. Chiang, M.-F. Wu, D.-C. Dai, Y.-S. Wen, J.-K. Wang and C.-T. Chen, Adv. Funct. Mater., 2005, 15, 231-238; $(g)$ T. M. Figueira-Duarte and K. Müllen, Chem. Rev., 2011, 111, 7260-7314.

2 (a) K. Yoshida, Y. Ooyama, S. Tanikawa and S. Watanabe, J. Chem. Soc., Perkin Trans. 2, 2002, 708-714; (b) Z. M. Hudson and S. Wang, Acc. Chem. Res., 2009, 42, 1584-1596; (c) Z. Chi, X. Zhang, B. Xu, X. Zhou, C. Ma, Y. Zhang, S. Liu and J. Xu, Chem. Soc. Rev., 2012, 41, 3878-3896; (d) L. K. Calderón Ortiz, H. Würfel, E. Täuscher, D. Weiß, E. Birckner, H. Görls and R. Beckert, Synthesis, 2014, 126-134.

3 (a) H. Mizuno, U. Haku, Y. Marutani, A. Ishizumi, H. Yanagi, F. Sasaki and S. Hotta, Adv. Mater., 2012, 24, 5744-5749; (b) O. Mhibik, T. Leang, A. Siove, S. Forget and S. Chénais, Appl. Phys. Lett., 2013, 102, 41112.

4 (a) N. Boens, W. Qin, N. Basaric, A. Orte, E. M. Talavera and J. M. Alvarez-Pe, J. Phys. Chem. A, 2006, 110, 9334-9343; (b) Q. A. Best, N. Sattenapally, D. J. Dyer, C. N. Scott and M. E. McCarroll, J. Am. Chem. Soc., 2013, 135, 13365-13370; (c) N. Marcotte and A. M. Brouwer, J. Phys. Chem. B, 2005, 109, 11819-11828.

5 (a) F. Riedel and S. Spange, J. Phys. Org. Chem., 2012, 25, 1261-1268; (b) K. Hofmann, S. Brumm, C. Mende, K. Nagel, A. Seifert, I. Roth, D. Schaarschmidt, H. Lang and S. Spange, New J. Chem., 2012, 36, 1655-1664; (c) A. Schade, N. Behme and S. Spange, Chem. - Eur. J., 2014, 20, 2232-2243.

6 (a) C. Reichardt, Solvents and Solvent Effects in Organic Chemistry, VCH, Weinheim, 2nd edn, 1988, and references therein; (b) C. Reichardt, Chem. Rev., 1994, 94, 2319-2358; (c) P. Müller, Pure Appl. Chem., 1994, 66, 1077-1079; (d) Y. Marcus, Chem. Soc. Rev., 1993, 22, 409-416; (e) V. Gutmann, Coord. Chem. Rev., 1976, 18, 225-240; ( $f$ ) V. Gutmann, The Donor-Acceptor Approach to Molecular Interactions, Plenum Press, New York, 1978.

7 M. J. Kamlet, J. L. M. Abboud, M. H. Abraham and R. W. Taft, J. Org. Chem., 1983, 48, 2877-2887.

8 (a) J. Catalán and C. Díaz, Liebigs Ann., 1997, 1941-1949; (b) J. Catalán and C. Díaz, Eur. J. Org. Chem., 1999, 885-891; (c) J. Catalán, C. Díaz, V. López, P. Pérez, J.-L. G. de Paz and J.-G. Rodríguez, Liebigs Ann., 1996, 1785-1794; (d) J. Catalán and H. Hopf, Chem. - Eur. J., 2004, 4694-4702; J. Catalán, J. Phys. Chem. B, 2009, 113, 5951-5960; (e) J. Catalán, J. Phys. Org. Chem., 2015, 28, 329-336. 
9 (a) V. Cavalli, D. C. da Silva, C. Machado, V. G. Machado and V. Soldi, J. Fluoresc., 2006, 16, 77-86; (b) M. El-Sayed, T. Blaudeck, F. Cichos and S. Spange, J. Photochem. Photobiol., A, 2007, 185, 44-50; (c) H. Akdas-Kilig, T. Roisnel, I. Ledoux and H. Le Bozec, New J. Chem., 2009, 33, 1470-1473; (d) I. Baraldi, E. Benassi, S. Ciorba, M. Šindler-Kulyk, I. Škorić and A. Spalletti, Chem. Phys., 2009, 361, 61-67; (e) D. Jana and B. K. Ghorai, Tetrahedron, 2012, 68, 7309-7316; $(f)$ Z. Seferoğlu, H. Ihmels and E. Şahin, Dyes Pigm., 2015, 113, 465-473.

10 L. Yin and J. Liebscher, Chem. Rev., 2007, 107, 133-173.

11 O. Reiser, Chem. Unserer Zeit, 2001, 2, 94-100.

12 N. Tyutyulkov, J. Fabian, A. Mehlhorn, F. Dietz and A. Tadjer, Polymethine Dyes - Structure and Properties, St. Kliment Ohridski University Press, Sofia, 1991.

13 C. Hansch, A. Leo and R. W. Taft, Chem. Rev., 1991, 91, 165-195.

14 J. Roger, F. Požgan and H. Doucet, Green Chem., 2009, 11, 425-432.

15 (a) A. Köhn and C. Hättig, J. Chem. Phys., 2003, 119, 5021-5036; (b) C. Hättig, J. Chem. Phys., 2003, 118,
7751-7761; (c) C. Hättig and F. Weigand, J. Chem. Phys., 2000, 113, 5154-5161.

16 F. Weigend and R. Ahlrichs, Phys. Chem. Chem. Phys., 2005, 7, 3297-3305.

17 F. Riedel, A. Oehlke and S. Spange, Z. Anorg. Allg. Chem., 2009, 635, 1335-1340.

18 J. Dobkowski, Z. R. Grabowski, J. Waluk, W. Kühnle, W. Rettig, C. Rullière, W. Yang, J. Adamus and J. Gebicki, Proc. Indiana Acad. Sci., 1992, 104, 143-152.

19 A. Filarowski, M. Kluba, K. Cieślik-Boczula, A. Koll, A. Kochel, L. Pandey, W. M. De Borggraeve, M. Van der Auweraer, J. Catalán and N. Boens, Photochem. Photobiol. Sci., 2010, 9, 996-1008.

20 Scilab Enterprises, Scilab: Free and Open Source software for numerical computation (OS, Version 5.4.1) [Software], 2012, Available from: http://www.scilab.org.

21 R. Ahlrichs, M. Bär, M. Häser, H. Horn and C. Kölmel, Chem. Phys. Lett., 1989, 162, 165-169.

22 O. Treutler and R. Ahlrichs, J. Chem. Phys., 1995, 102, 346-354. 